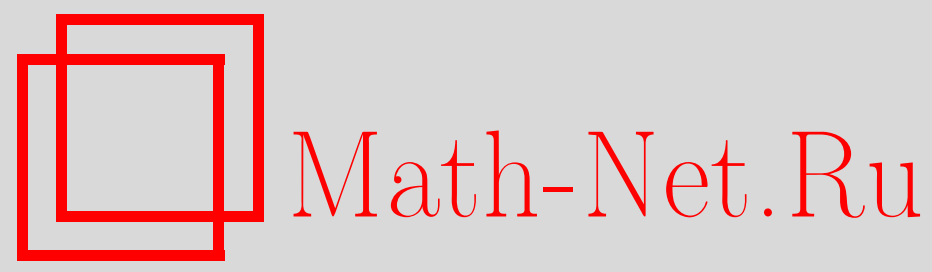

В. В. Шпаков, Н. Т. Воробьев, Локальные факторизации нелокальных классов Фиттинга, Дискрет. матем., 2008, том 20, выпуск 3, 111-118

DOI: https://doi.org/10.4213/dm1018

Использование Общероссийского математического портала Math-Net.Ru подразумевает, что вы прочитали и согласны с пользовательским соглашением http://www . mathnet.ru/rus/agreement

Параметры загрузки:

IP: 3.89 .197 .203

26 апреля 2023 г., 08:15:39 


\title{
Локальные факторизации нелокальных классов Фиттинга
}

\author{
() 2008 г. В. В. Шпаков, Н. Т. Воробьев
}

\begin{abstract}
В работе выявлены общие закономерности построения локальных произведений классов Фиттинга посредством нелокальных множителей, которые определяются при помощи наименьших элементов секции Локетта. Найден простой метод построения локальных классов Фиттинга, факторизуемых нелокальными классами Фиттинга некоторых классов $\pi$-групп и $\pi^{\prime}$-групп. При этом, применяя класс $\mathfrak{B}$, мы упрощаем процедуру построения нелокального множителя в локальном произведении. Установлено, что при построении локального произведения нелокальным множителем для подходящего множества простых $\pi$ является класс Фиттинга вида $\mathfrak{B}_{*} \mathfrak{S}_{\pi}$, где $\mathfrak{B}_{*}$ - наименьший элемент секции Локетта класса $\mathfrak{B}$. В работе рассматриваются только конечные разрешимые группы.
\end{abstract}

\section{1. Введение}

Произведением классов Фиттинга [1] $\mathfrak{F}$ и $\mathfrak{H}$ называют класс всех тех групп $G$, факторгруппы по $\mathfrak{F}$-радикалу которых являются $\mathfrak{5}$-подгруппами. Хорошо известно, что произведение двух классов Фиттинга снова является классом Фиттинга и операция умножения классов Фиттинга ассоциативна (см, например, IX.1.12 в [1]). Среди произведений классов Фиттинга наиболее известны своими приложениями локальные произведения, то есть такие произведения, которые являются локальными классами Фиттинга. В [2] было доказано, что произведение двух любых локальных классов Фиттинга является локальным.

Напомним, что всякое отображение $f$ множества $P$ всех простых чисел в множество классов Фиттинга называют функцией Хартли или Н-функцией [3]. Класс Фиттинга $\mathfrak{F}$ называют локальным, если существует такая Н-функция $f$, что

$$
\mathfrak{F}=\overleftarrow{S}_{\pi(\mathfrak{F})} \cap\left(\bigcap_{p \in \pi(\mathfrak{F})} f(p) \Re_{p} \widetilde{S}_{p^{\prime}}\right) .
$$

Построение примеров локальных классов Фиттинга и локальных формаций, факторизуемых нелокальными множителями, было осуществлено впервые Н. Т. Воробьевым, А. Н. Скибой (см. [4]) и В. А. Ведерниковым (см. [5]), Н. Т. Воробьевым (см. [6]) соответственно, и тем самым было получено положительное решение вопросов 11.25 а) и 9.58 из [7].

В настоящей работе выявлены общие закономерности построения локальных произведений классов Фиттинга посредством нелокальных множителей, которые определяются при помощи наименьших элементов секции Локетта [8] (см. Х.1.12(b) и X.1.16 в [1]). 
Прежде всего, найден простой метод построения локальных классов Фиттинга, факторизуемых нелокальными классами Фиттинга некоторых классов $\pi$-групп и $\pi^{\prime}$-групп (теорема 1, случай 1). При этом, в отличие от [4], мы не используем громоздкую конструкцию класса Фиттинга $\mathfrak{B}$, который был определен в [9]. Кроме того, применяя класс $\mathfrak{B}$, мы упрощаем, по сравнению с [4], процедуру построения нелокального множителя в локальном произведении. Установлено, что при построении локального произведения, отличного от произведения примера [4], нелокальным множителем для подходящего множества простых $\pi$ является класс Фиттинга вида $\mathfrak{B}_{*} \mathfrak{\subseteq}_{\pi}$, где $\mathfrak{B}_{*}-$ наименьший элемент секции Локетта класса $\mathfrak{B}$. Заметим также, что в дополнение к [4], нами показано, что каждый из множителей локального произведения, определяется полулокально. В работе рассматриваются только конечные разрешимые группы.

Определения и обозначения при необходимости можно найти в $[1,10]$.

\section{2. Предварительные сведения}

Класс групп $\mathfrak{F}$ называется классом Фиттинга [1], если $\mathfrak{F}$ замкнут относительно взятия нормальных подгрупп и произведения нормальных $\mathfrak{F}$-подгрупп. Если $\mathfrak{F}-$ непустой класс Фиттинга, то подгруппа $G_{\mathfrak{F}}$ группы $G$ называется $\mathfrak{F}$-радикалом группы $G$, если она является наибольшей из нормальных подгрупп $G$, принадлежащих $\mathfrak{F}$.

Приведем в качестве лемм некоторые известные свойства $\mathfrak{F}$-радикалов и произведений классов Фиттинга, которые мы будем использовать.

Класс групп $\mathfrak{F}$ называют гомоморфом [10], если каждая факторгруппа любой группы из $\mathfrak{F}$ также принадлежит $\mathfrak{F}$.

Лемма 1 (лемма 2.1 в [11]). Пусть $\mathfrak{F}$ - непустой класс Фиттинга. Тогда справедливь следуюшие утверждения:

(1) $\mathfrak{F} \subseteq \mathfrak{F} \mathfrak{H}$ для любого класса Фиттинга $\mathfrak{H} \neq \varnothing$,

(2) если класс Фиттинга $\mathfrak{H}-$ гомоморф, то $\mathfrak{H} \subseteq \mathfrak{F} \mathfrak{H}$,

(3) если $\left\{\mathfrak{H}_{i} \mid i \in I\right\}$ - непустое множество классов Фиттинга, то

$$
\bigcap_{i \in I} \mathfrak{F} \mathfrak{S}_{i}=\mathfrak{F}\left(\bigcap_{i \in I} \mathfrak{S}_{i}\right) .
$$

Лемма 2 ([2]). Произведение двух любых локальных классов Фиттинга является локальHolм.

Гомоморф $\mathfrak{F}$ является насыщенным [10], если из условия $G / \Phi(G) \in \mathfrak{F}$ следует включение $G \in \mathfrak{F}$. Класс групп $\mathfrak{F}$ называют радикальным гомоморфом [10], если он является одновременно гомоморфом и классом Фиттинга.

Напомним, что для любого класса Фиттинга $\mathfrak{F}$ Локетт [8] определил класс $\mathfrak{F}^{*}$ как наименьший из классов Фиттинга, содержащий $\mathfrak{F}$ такой, что для всех групп $G$ и $H$ справедливо равенство $(G \times H)_{\mathfrak{F}} * G_{\mathfrak{F}} * H_{\mathfrak{F}}$, и класс $\mathfrak{F} *$ как пересечение всех таких классов Фиттинга $\mathfrak{X}$, для которых $\mathfrak{X}^{*}=\mathfrak{F}^{*}$. Класс Фиттинга $\mathfrak{F}$ называется классом Локетта, если $\mathfrak{F}=\mathfrak{F}^{*}$.

Приведем в виде леммы известные свойства операторов Локетта ${ }^{*}$ и *.

Лемма 3 (X.1.2, IX.1.8 в [1]; [8], [11]). Для любого непустого класса Фиттинга ₹ справедливы следующие утверждения: 
(1) действия операторов * $u$ * на класс $\mathfrak{F}$ характеризуют следуюшче соотношения:

$$
\mathfrak{F}_{*}=\left(\mathfrak{F}_{*}\right)_{*}=\left(\mathfrak{F}^{*}\right)_{*} \subseteq \mathfrak{F} \subseteq \mathfrak{F}^{*}=\left(\mathfrak{F}_{*}\right)^{*}=\left(\mathfrak{F}^{*}\right)^{*}
$$

(2) для любого непустого класса Фиттинга $\mathfrak{H}$ справедливо равенство

$$
\left(\mathfrak{F} \cap \mathfrak{S}^{*}=\mathfrak{F}^{*} \cap \mathfrak{S}^{*}\right.
$$

(3) если $\mathfrak{F}-$ гомоморф, то $\mathfrak{F}^{*}=\mathfrak{F}$;

(4) если $\mathfrak{5}$ - радикальный насыщенный гомоморф, то

$$
(\mathfrak{F} \mathfrak{S})^{*}=\mathfrak{F}^{*} \mathfrak{H}
$$

Лемма 4 ([11]). Каждый локальный класс Фиттинга является классом Локетта.

Класс Фиттинга $\mathfrak{F}$ называют нормальным [1], если $\mathfrak{F}$-радикал группы $G$ является $\mathfrak{F}$-максимальной подгруппой группы $G$ для любой группы $G$. Заметим, что пересечение любого множества неединичных нормальных классов Фиттинга является неединичным нормальным классом Фиттинга. Наименьший неединичный нормальный класс Фиттинга обозначают $\mathfrak{S}_{*}$.

Лемма 5 (Х.5.32 в [1]). Если р и q-различные простые числа, то $\mathfrak{\Re}_{p} \mathfrak{N}_{q} \nsubseteq_{\mathbb{S}_{*}}$.

Напомним, что за некоторыми классами закреплены стандартные обозначения: $\subseteq$ класс всех разрешимых групп; $\mathfrak{S}_{\pi}-$ класс всех разрешимых $\pi$-групп; $\mathfrak{\Re}-$ класс всех нильпотентных групп; $\mathfrak{\Re}_{\pi}-$ класс всех нильпотентных $\pi$-групп.

Лемма 6 ([8]). Если $\mathfrak{F}$ - непустой класс Фиттинга, то следующие утверждения равносильнье:

(1) $\mathfrak{F}$ - нормальный класс Фиттинга;

(2) $\mathfrak{F} \mathfrak{N}=\mathbb{S}$;

(3) $\mathfrak{F}^{*}=\mathfrak{\subseteq}$.

Класс Фиттинга $\mathfrak{F}$, для которого $\mathfrak{F}_{*}=\mathfrak{F}^{*} \cap \mathfrak{S}_{*}$, называют удовлетворяющим гипотезе Локетта [11] или $\mathfrak{L}$-классом.

Лемма 7 ([11]). Каждый локальный класс Фиттинга является -классом.

Класс Фиттинга $\mathfrak{F}$ называют $\pi$-насыщенным [12], если $\mathfrak{F} \Im_{\pi^{\prime}}=\mathfrak{F}$, где $\varnothing \neq \pi \subseteq P$ и $\Im_{\pi^{\prime}}-$ класс всех разрешимых $\pi^{\prime}$-групп.

\section{3. Класс $R_{\pi}(\mathfrak{F})$ и его свойства}

Пусть

$$
\pi=\operatorname{Supp}(f)=\{p \in P: f(p) \neq \varnothing\}
$$

- носитель Н-функции $f$. Тогда, следуя [12], положим

$$
S L R(f)=\bigcap_{p \in \pi} f(p) \Im_{p^{\prime}}
$$


и класс Фиттинга $\mathfrak{F}$ определяется полулокально [13], если $\mathfrak{F}=S L R(f)$ для некоторой Н-функции $f$. Если $\pi=\varnothing$, то положим $\operatorname{SLR}(f)=\varnothing$.

Критерий класса Фиттинга, определяемого полулокально, дает следующее утверждение.

Лемма 8. Класс Фиттинга $\mathfrak{F}$ определяется полулокально тогда и только тогда, когда $\mathfrak{F}-\pi$-насыщен для некоторого $\pi, \varnothing \subset \pi \subseteq P$.

Доказательство. Пусть $\varnothing \subset \pi \subseteq P$ и $\mathfrak{F}-\pi$-насыщенный класс Фиттинга. Тогда $\mathfrak{F}_{\pi^{\prime}}=\mathfrak{F}$. Построим Н-функцию $f$ следующим образом:

$$
f(p)= \begin{cases}\mathfrak{F}, & \text { если } p \in \pi, \\ \varnothing, & \text { если } p \in \pi^{\prime}\end{cases}
$$

Покажем, что $f$ определяет полулокально класс Фиттинга $\mathfrak{F}$. Пусть

$$
\operatorname{SLR}(f)=\bigcap_{p \in \pi} f(p) \Im_{p^{\prime}}
$$

где $\pi=\operatorname{Supp}(f)$. Для всех $p \in \pi$ значения Н-функции $f(p)=\mathfrak{F}$, поэтому

$$
\operatorname{SLR}(f)=\bigcap_{p \in \pi} \mathfrak{F} \widetilde{S}_{p^{\prime}}
$$

Следовательно, по утверждению 3 леммы 1

$$
\operatorname{SLR}(f)=\mathfrak{F}\left(\bigcap_{p \in \pi} \mathfrak{S}_{p^{\prime}}\right)=\mathfrak{F} \mathfrak{S}_{\pi^{\prime}}=\mathfrak{F},
$$

и $\mathfrak{F}$ является классом Фиттинга, определяемым полулокально.

Пусть теперь $\mathfrak{F}$ - класс Фиттинга, определяемый полулокально. Тогда существует Н-функция $f$ с носителем $\pi$ такая, что

$$
\mathfrak{F}=\bigcap_{p \in \pi} f(p) \widetilde{\Im}_{p^{\prime}}
$$

Покажем, что

$$
\mathfrak{F} \mathfrak{\Im}_{\pi^{\prime}}=\mathfrak{F}
$$

Так как

$$
\mathfrak{F}_{\pi^{\prime}}=\left(\bigcap_{p \in \pi} f(p) \Im_{p^{\prime}}\right) \Im_{\pi^{\prime}},
$$

ввиду утверждения 3 леммы 1

$$
\mathfrak{F}_{\pi^{\prime}}=\bigcap_{p \in \pi}(f(p)) \Im_{p^{\prime}} \Im_{\pi^{\prime}} .
$$

Но $p \in \pi$, и поэтому $\pi^{\prime} \subseteq p^{\prime}$. Следовательно, $\mathfrak{\subseteq}_{\pi^{\prime}} \subseteq \mathfrak{S}_{p^{\prime}}$ для всех простых $p \in \pi$. Кроме того, $\Im_{p^{\prime}} \Im_{\pi^{\prime}}=\Im_{p^{\prime}}$. Значит,

$$
\mathfrak{F} \mathfrak{S}_{\pi^{\prime}}=\bigcap_{p \in \pi} f(p)\left(\mathbb{S}_{p^{\prime}} \mathfrak{\Im}_{\pi^{\prime}}\right)=\bigcap_{p \in \pi} f(p) \mathfrak{S}_{p^{\prime}}=\mathfrak{F}
$$

Лемма доказана. 
Определение 1. Пусть $\pi \subseteq P$. Определим класс групп $R_{\pi}(\mathfrak{F})$ следующим образом:

$$
G \in R_{\pi}(\mathfrak{F}) \Longleftrightarrow G_{\pi} \subseteq G_{\mathfrak{F}} .
$$

Если $\mathfrak{F}=\varnothing$, то положим $R_{\pi}(\mathfrak{F})=\varnothing$. В случае, когда $\pi=\varnothing$ и $\pi=P$, положим $R_{\varnothing}(\mathfrak{F})=\mathfrak{S}$ и $R_{P}(\mathfrak{F})=\mathfrak{F}$ соответственно.

Заметим, что если $\mathfrak{F}$ - нормальный класс Фиттинга, то $R_{\pi}(\mathfrak{F})=L_{\pi}(\mathfrak{F})$, где $L_{\pi}(\mathfrak{F})$ - класс всех тех групп $G, \mathfrak{F}$-инъекторы которых содержат холлову $\pi$-подгруппу $G$ (см. IX.1.14 в [1]).

Лемма 9. Если $\mathfrak{F}-$ класс Фиттинга $и \pi \subseteq P$, то $R_{\pi}(\mathfrak{F})=\mathfrak{F}_{\pi^{\prime}}$ является $\pi$-насыщзенным (полулокальным) классом Фиттинга. В частности, если $\mathfrak{F}-$ локальный класс Фиттинга, то $R_{\pi}(\mathfrak{F})$ - локальный класс Фиттинга.

Доказательство. Пусть $G \in R_{\pi}(\mathfrak{F})$. Тогда $G_{\pi} \subseteq G_{\mathfrak{F}}$, и значит, $G / G_{\mathfrak{F}}$ есть $\pi^{\prime}$-группа. Так как $G_{\mathfrak{F}} \in \mathfrak{F}$ и $G / G_{\mathfrak{F}} \in \mathbb{S}_{\pi^{\prime}}$, то $G \in \mathfrak{F} \widetilde{\subseteq}_{\pi^{\prime}}$. Следовательно, $R_{\pi}(\mathfrak{F}) \subseteq \mathfrak{F}_{\pi^{\prime}}$. Пусть $H \in \mathfrak{F}_{\pi^{\prime}}$. Тогда $H / H_{\mathfrak{F}} \in \mathfrak{S}_{\pi^{\prime}}$ и значит $H_{\pi} \subseteq H_{\mathfrak{F}}$. Следовательно, $H \in R_{\pi}(\mathfrak{F})$ и $\mathfrak{F}_{\pi^{\prime}} \subseteq R_{\pi}(\mathfrak{F})$. Поэтому $R_{\pi}(\mathfrak{F})=\mathfrak{F}_{\pi^{\prime}}$ является классом Фиттинга. Так как

$$
R_{\pi}(\mathfrak{F}) \mathfrak{S}_{\pi^{\prime}}=\left(\mathfrak{F} \mathfrak{\Im}_{\pi^{\prime}}\right) \mathfrak{\Im}_{\pi^{\prime}}=\mathfrak{F}\left(\mathfrak{S}_{\pi^{\prime}} \mathfrak{\Im}_{\pi^{\prime}}\right)=\mathfrak{F} \mathfrak{\Im}_{\pi^{\prime}}=R_{\pi}(\mathfrak{F}),
$$

то $R_{\pi}(\mathfrak{F})-\pi$-насыщенный класс Фиттинга. Пусть $\mathfrak{F}-$ локальный класс Фиттинга. Так как $\mathfrak{\Im}_{\pi^{\prime}}-$ локальный класс Фиттинга, по лемме $2 R_{\pi}(\mathfrak{F})=\mathfrak{F}_{\pi^{\prime}}-$ локальный класс Фиттинга. Лемма доказана.

Заметим, что в общем случае класс Фиттинга $R_{\pi}(\mathfrak{F})$ нелокален. Это подтверждает следующий пример.

Пример 1. Пусть $\mathfrak{F}=\mathfrak{S}_{*}-$ наименьший нормальный класс Фиттинга и $\varnothing \neq \pi \subset P$. Покажем, что в этом случае класс $R_{\pi}(\mathfrak{F})$ не является локальным. Предположим, что $R_{\pi}(\mathfrak{F})$ - локальный класс Фиттинга. Из леммы 6, ввиду нормальности класса $\mathfrak{F}$, следует, что $\mathfrak{F} \mathfrak{N}=\mathbb{S}$. Так как $\mathfrak{F} \subseteq R_{\pi}(\mathfrak{F})$, то $\mathfrak{S}=\mathfrak{F} \mathfrak{N} \subseteq R_{\pi}(\mathfrak{F}) \mathfrak{N} \subseteq \mathfrak{S}$. Значит, $R_{\pi}(\mathfrak{F}) \mathfrak{N}=\mathfrak{S}$ и по лемме $6 R_{\pi}(\mathfrak{F})$ - нормальный класс Фиттинга. По лемме $9, R_{\pi}(\mathfrak{F})=\mathfrak{F} \mathfrak{\Im}_{\pi^{\prime}}$, по утверждению 4 леммы $3\left(R_{\pi}(\mathfrak{F})\right)^{*}=\left(\mathfrak{F}_{\mathbb{\pi}^{\prime}}\right)^{*}=\mathfrak{F}^{*} \mathfrak{S}_{\pi^{\prime}}$. Но ввиду леммы 6 и утверждения 1 леммы $3 \mathfrak{F}^{*}=\mathfrak{S}$ и $\left(R_{\pi}(\mathfrak{F})\right)^{*}=\mathfrak{S}$. Теперь, ввиду локальности $R_{\pi}(\mathfrak{F})$, по лемме 4 $R_{\pi}(\mathfrak{F})$ - класс Локетта, и следовательно, $R_{\pi}(\mathfrak{F})=\mathfrak{S}$.

Пусть теперь $p$ и $q$ - такие простые числа, что $p \mid(q-1), G=D_{q^{n}}^{n}$ - монолитическая группа с нормальной абелевой силовской $q$-подгруппой экспоненты $q^{n}$ и циклической силовской $q^{\prime}$-подгруппой порядка $p$. Пусть $\pi=\pi(G)$. Тогда $G_{\pi}=G$ и, ввиду результата Бергера (см. свойство 3 из [14]), группа $G \notin \mathfrak{S}_{*}$. Следовательно, $G_{\varsigma_{*}} \subset G$. Значит, $G \notin R_{\pi}(\mathfrak{F})$ и $R_{\pi}(\mathfrak{F}) \neq \mathfrak{S}$. Полученное противоречие доказывает, что класс Фиттинга $R_{\pi}(\mathfrak{F})$ нелокален.

\section{4. Локальные факторизации}

Лемма 10. Если $\pi \subset P u|\pi| \geqslant 2$, то произведение $\left(\Im_{\pi}\right)_{*} \Im_{\pi^{\prime}}$ не является классом Локетта.

Доказательство. Предположим, что $\left(\mathfrak{S}_{\pi}\right)_{*} \mathfrak{\subseteq}_{\pi^{\prime}}-$ класс Локетта. Тогда, согласно утверждениям 1 и 4 леммы 3,

$$
\left(\Im_{\pi}\right)_{*} \Im_{\pi^{\prime}}=\left(\left(\Im_{\pi}\right)_{*} \Im_{\pi^{\prime}}\right)^{*}=\left(\left(\Im_{\pi}\right)_{*}\right) * \Im_{\pi^{\prime}}=\left(\Im_{\pi}\right)^{*} \widetilde{S}_{\pi^{\prime}}=\Im_{\pi} \Im_{\pi^{\prime}}
$$


Класс Фиттинга $\Im_{\pi}=L R(f)$ для Н-функции $f$ такой, что

$$
f(p)= \begin{cases}\Im_{\pi}, & \text { если } p \in \pi, \\ \varnothing, & \text { если } p \in \pi^{\prime} .\end{cases}
$$

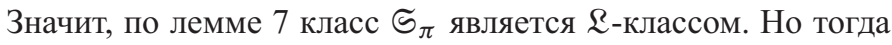

$$
\left(\widetilde{S}_{\pi}\right)_{*} \widetilde{S}_{\pi^{\prime}}=\left(\widetilde{S}_{\pi} \cap \widetilde{S}_{*}\right) \widetilde{S}_{\pi^{\prime}}=\widetilde{S}_{\pi} \widetilde{S}_{\pi^{\prime}} \cap \mathfrak{S}_{*} \widetilde{S}_{\pi^{\prime}}
$$

Следовательно, $\mathfrak{S}_{\pi} \mathfrak{S}_{\pi^{\prime}}=\mathfrak{S}_{\pi} \mathfrak{S}_{\pi^{\prime}} \cap \mathfrak{S}_{*} \mathfrak{S}_{\pi^{\prime}}$ и поэтому $\mathfrak{S}_{\pi} \mathfrak{S}_{\pi^{\prime}} \subseteq \mathfrak{S}_{*} \mathfrak{S}_{\pi^{\prime}}$. Значит,

$$
\mathfrak{S}_{\pi} \subseteq \mathfrak{S}_{*} \mathfrak{S}_{\pi} \cap \mathfrak{S}_{*} \mathfrak{S}_{\pi^{\prime}}=\mathfrak{S}_{*}\left(\mathfrak{S}_{\pi} \cap \mathfrak{S}_{\pi^{\prime}}\right)
$$

Таким образом, $\mathfrak{\subseteq}_{\pi} \subseteq \mathfrak{S}_{*}$. Но для $|\pi| \geqslant 2$ это невозможно по лемме 5. Полученное противоречие показывает, что класс Фиттинга $\left(\mathfrak{S}_{\pi}\right)_{*} \mathfrak{S}_{\pi^{\prime}}$ не является классом Локетта. Лемма доказана.

Лемма 11. Если $\mathfrak{F}$ - класс Локетта, то класс $R_{\pi}(\mathfrak{F})$ является классом Локетта.

Доказательство. По лемме $9 R_{\pi}(\mathfrak{F})=\mathfrak{F}_{\pi^{\prime}}$. Следовательно, $\left(R_{\pi}(\mathfrak{F})\right)^{*}=\left(\mathfrak{F}_{\pi^{\prime}}\right)^{*}$. Тогда по утверждению 4 леммы $3\left(\mathfrak{F} \mathfrak{S}_{\pi^{\prime}}\right)^{*}=\mathfrak{F}^{*} \mathfrak{S}_{\pi^{\prime}}$. Но $\mathfrak{F}-$ класс Локетта, и поэтому $\mathfrak{F}^{*}=\mathfrak{F}$. Значит $\mathfrak{F}^{*} \mathfrak{S}_{\pi^{\prime}}=\mathfrak{F}_{\pi^{\prime}}$. Получаем, что $\left(R_{\pi}(\mathfrak{F})\right)^{*}=R_{\pi}(\mathfrak{F})$ и $R_{\pi}(\mathfrak{F})-$ класс Локетта. Лемма доказана.

Заметим, что ввиду результата Косси [14] тривиальный локальный класс Фиттинга ऽ всегда можно представить в виде локального произведения $\mathfrak{\subseteq}=\mathfrak{F}_{*}$ для любого неединичного нормального класса Фиттинга $\mathfrak{F} \neq \mathfrak{S}$. При этом ввиду лемм 4 и 6 легко видеть, что каждый из множителей $\mathfrak{F}$ и $\mathfrak{S}_{*}$ нелокален и не является формацией.

Для построения локальных произведений (отличных от )ㅜ нелокальных ненормальных классов Фиттинга, которые не являются формациями, мы будем, в частности, использовать класс Фиттинга $\mathfrak{B}$, определенный Бергером и Косси в [9].

Напомним, что построение класса $\mathfrak{B}$ редуцируется к нахождению некоторой группы $X$, одновременно содержащейся в классах $\mathfrak{B}$ и $\mathfrak{S}_{*}$, но не принадлежащей классу $\mathfrak{B}_{*}$. Для этой цели используется описание представлений экстраспециальных $p$-групп над произвольным полем характеристики, не равной $p$ (см. [1], с. 166-168). Пусть теперь $p=3$ и $R$ - экстраспециальная группа порядка 27 экспоненты 3. Тогда по утверждению (ii) теоремы 9.16 в [1] $R$ имеет точный абсолютно неприводимый модуль $W$ размерности 3 над полем $G F(7)$ (существование такого модуля доказано в [9]). Пусть $Y=[W] R$. Обозначим через $A$ группу автоморфизмов группы $R$. Пусть $B=C_{A}(Z(R)), Q-$ подгруппа кватернионов группы $B$ и $X=Z(Q) Y$.

Тогда введем класс

$$
\mathfrak{M}=\left(G \mid O^{2^{\prime}}\left(G / O_{\{2,3\}}(G)\right) \in S_{n} D_{o}(X)\right),
$$

где $D_{o}(X)$ - класс всех конечных прямых произведений изоморфных копий группы $X$. Введем обозначение $\mathfrak{B}=\mathfrak{M} \cap \mathfrak{S}_{7} \mathfrak{S}_{3} \mathfrak{S}_{2}$. Согласно теореме 4.5 в [9], класс $\mathfrak{B}$ является классом Локетта и не является классом Фишера. Кроме того, $\mathfrak{B}$ обладает свойством, приведенным в следующем утверждении.

Лемма 12 (теорема 4.4 в [9])). Если $\Im_{*}-$ наименьший нормальный класс Фиттинга, то $X \in\left(\mathfrak{B} \cap \mathfrak{S}_{*}\right) \backslash \mathfrak{B}_{*}$. 
Лемма 13. Если $\pi(X) \subseteq \sigma \subset P$, то класс Фиттинга $R_{\sigma}\left(\mathfrak{B}_{*}\right)$ не является классом Локетта.

Доказательство. Предположим, что $\left(R_{\sigma}\left(\mathfrak{B}_{*}\right)\right)^{*}=R_{\sigma}\left(\mathfrak{B}_{*}\right)$. Поскольку по утверждению 1 леммы $3\left(\mathfrak{B}_{*}\right)^{*}=\mathfrak{B}^{*}$, применяя лемму 9 и утверждение 4 леммы 3 , получаем равенство

$$
\left(R_{\sigma}\left(\mathfrak{B}_{*}\right)\right)^{*}=\left(\mathfrak{B}_{*} \mathfrak{S}_{\sigma^{\prime}}\right)^{*}=\left(\mathfrak{B}_{*}\right)^{*} \mathfrak{S}_{\sigma^{\prime}}=\mathfrak{B}^{*} \mathfrak{S}_{\sigma^{\prime}}=R_{\sigma}\left(\mathfrak{B}^{*}\right) .
$$

Но по лемме $12 \mathfrak{B}$ является классом Локетта. Следовательно, $R_{\sigma}\left(\mathfrak{B}_{*}\right)=R_{\sigma}(\mathfrak{B})$. Согласно лемме $12, X \in \mathfrak{B}$, поэтому $X=X_{\sigma}=X_{\mathfrak{B}}$. Значит, $X \in R_{\sigma}(\mathfrak{B})=R_{\sigma}\left(\mathfrak{B}_{*}\right)$. Но тогда $X=X_{\sigma} \subseteq X_{\mathfrak{B}_{*}} \subseteq X$. Отсюда следует, что $X=X_{\mathfrak{B}_{*}}$ и $X \in \mathfrak{B}_{*}$. Получено противоречие. Следовательно, $R_{\sigma}\left(\mathfrak{B}_{*}\right)$ не является классом Локетта. Лемма доказана.

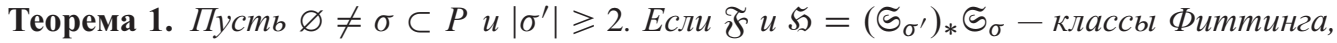
то произведение $\mathfrak{F} 5$ локально, а каждый из множителей $\mathfrak{F}$ и $\mathfrak{5}$ нелокален, произведение определяется полулокально и не является формаџией для каждого из следующих значений $\mathfrak{F}$ :

(1) $\mathfrak{F}=\left(\widetilde{\subseteq}_{\sigma}\right)_{*} \mathfrak{S}_{\sigma^{\prime}}$ для $|\sigma| \geqslant 2$;

(2) $\mathfrak{F}=R_{\sigma}\left(\mathfrak{B}_{*}\right)$ для $\sigma \supseteq \pi(X)$.

Доказательство. Пусть $\mathfrak{F}=\left(\mathfrak{S}_{\sigma}\right)_{*} \mathfrak{S}_{\sigma^{\prime}}$, причем множества простых $\sigma$ и $\sigma^{\prime}$ таковы, что $|\sigma| \geqslant 2$ и $\left|\sigma^{\prime}\right| \geqslant 2$. Так как $\mathfrak{F} \mathfrak{S}_{\sigma^{\prime}}=\mathfrak{F}$ и $\mathfrak{S} \mathfrak{S}_{\sigma}=\mathfrak{H}$, классы $\mathfrak{F}$ и $\mathfrak{H}$ по лемме 8 определяются полулокально. Учитывая лемму 10 , заключаем, что классы $\mathfrak{F}$ и $\mathfrak{5}$ не являются классами Локетта. Следовательно, $\mathfrak{F}$ и $\mathfrak{5}$ ввиду утверждения 3 леммы 3 не являются формациями.

Теперь с учетом утверждения 1 леммы 3

$$
\mathfrak{F} \mathfrak{H}=\left(\mathbb{S}_{\sigma}\right)_{*} \mathfrak{S}_{\sigma^{\prime}}\left(\mathbb{S}_{\sigma^{\prime}}\right)_{*} \mathbb{S}_{\sigma}=\left(\mathbb{S}_{\sigma}\right)_{*} \mathbb{S}_{\sigma^{\prime}} \mathbb{S}_{\sigma} .
$$

Пусть $f$ такая Н-функция, что

$$
f(p)= \begin{cases}\left(\Im_{\sigma}\right)_{*} \Im_{\sigma^{\prime}}, & \text { если } p \in \sigma^{\prime} \\ \left(\Im_{\sigma}\right)_{*} \Im_{\sigma^{\prime}} \Im_{\sigma}, & \text { если } p \in \sigma\end{cases}
$$

Покажем, что $\mathfrak{F} \mathfrak{5}=L R(f)$. Действительно,

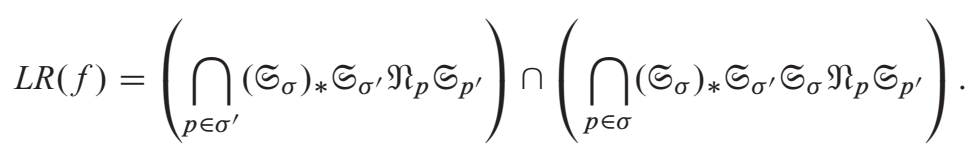

Так как $\mathfrak{S}_{\sigma^{\prime}} \mathfrak{M}_{p}=\mathfrak{S}_{\sigma^{\prime}}$ для всех $p \in \sigma^{\prime}$ и $\mathfrak{S}_{\sigma} \mathfrak{M}_{p}=\mathfrak{S}_{\sigma}$ для всех $p \in \sigma$, то

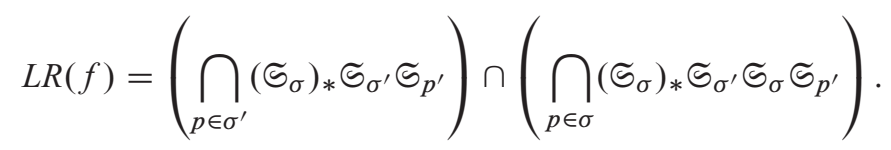

Используя утверждение 3 леммы 1, получаем, что

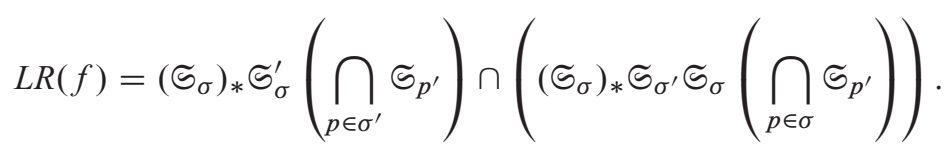


Учитывая, что

$$
\bigcap_{p \in \sigma^{\prime}} \Im_{p^{\prime}}=\Im_{\sigma}, \quad \bigcap_{p \in \sigma} \Im_{p^{\prime}}=\Im_{\sigma^{\prime}},
$$

находим, что

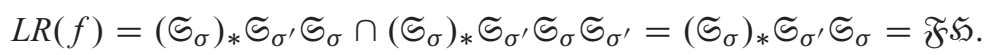

Итак, в случае $1 \mathfrak{F} \mathfrak{5}$ - локальное произведение классов Фиттинга $\mathfrak{F}$ и $\mathfrak{5}$.

В случае, когда $\mathfrak{F}=R_{\sigma}\left(\mathfrak{B}_{*}\right)$, теорема доказывается аналогично с учетом лемм 8,9 , 11 и 12. Теорема доказана.

\section{Список литературы}

1. Doerk K., Hawkes T., Finite solvable groups. Walter de Gruyter, Berlin, 1992.

2. Воробьев Н. Т., Локальные произведения классов Фиттинга. Весці АН БССР. Сер. фiз.-мат. навук (1991) №6, 28-32.

3. Воробьев Н. Т., О предположении Хоукса для радикальных классов. Сибирский матем. журнал (1996) 37, №5, 1296-1302.

4. Воробьев Н. Т., Скиба А. Н., Локальные произведения нелокальных классов Фиттинга. Bonpocbl алгебры (1995) 8, 55-58.

5. Ведерников В. А., О локальных формациях конечных групп. Матем. заметки (1989) 46, №6, 32-37.

6. Vorob'ev N. T., On the factorization of local and non-local products of finite groups of non-local formations. In: Proc. 7th Reg. Sci. Sess. Math., Sect. Algebra Number Theory, Kalsk/Pol., 1990, pp. 9-13.

7. Коуровская тетрадь (нерешенные вопросы теории групп), издание 11. ИМ РАН, Новосибирск, 1990 (задачи 11.25 а) и 9.58).

8. Lockett P., The Fitting class $\mathfrak{F}^{*}$. Math. Z. (1974) 137, №2, 131-136.

9. Berger R., Cossey J., An example in theory of normal Fitting classes. Math. Z. (1978) 154, №1, 573-578.

10. Шеметков Л. А., Формачии конечных групn. Наука, Москва, 1978.

11. Воробьев Н. Т., О радикальных классах конечных групп с условием Локетта. Матем. заметки (1988) 43, №2, 161-168.

12. Воробьев Н. Т., О локальных радикальных классах. Вопросы алгебры (1986) 2, 41-50.

13. Vorob'ev N. T., Gaschutz's method in the theory of Fitting classes of finite soluble groups. Изв. Гомельского ун-та. Вопросы алгебры (2000) 3, №16, 155-166.

14. Berger T. K., More normal Fitting classes of finite solvable groups. Math. Z. (1976) 151, №1, 1-3.

15. Cossey J., Products of Fitting classes. Math. Z. (1975) 141, №3, 289-295.

Статья поступила 4.06.2007. 